\title{
A Study on the Architecture of Virtual Reality System for Art Education
}

\author{
SangHun Nam ${ }^{1}$, SangHwa Lee ${ }^{2}$ and JungYoon $\mathrm{Kim}^{3 *}$ \\ ${ }^{1}$ Department of Newmedia, Seoul Media Institute of Technology, \\ Seoul, South Korea \\ ${ }^{2}$ Scool of Manhwa Contents at Chungkang College of Cultural of Industries, \\ Majang-myeon Gyeonggi-Do, South Korea \\ ${ }^{3}$ Graduate School of Game, Gachon University, 1342 Seongnam Daero, Sujeong- \\ Gu, Seongnam-Si, Gyeonggi-Do, South Korea, 461-701

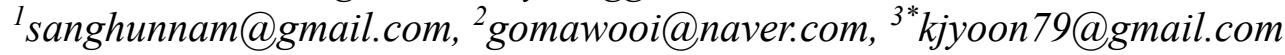

\begin{abstract}
Virtual reality (VR) industry has been growing explosively as one of the versatile technologies that may lead ICT industry of next generation. As this technology develops at a fast speed, educational environments for children and educators also change very quickly. However, one of the main problems with virtual reality as a learning tool for art is that there are hardly any theories or models upon which to build a foundation and justify its application. In this study, a possibility of art education applying VR technology that may express a sense of space and interaction for the users was analyzed. As interactive media art works using VR provides a new sense of space, a new meaning that was not experienced by the audience in the past could be delivered. Delivery mode of a new meaning could be also used for art education effectively. Art work applying VR technology was observed and based on this result, an effect of VR technology on art education was researched. The art environments support interaction between educators and learners and enable multiple simultaneous participants to access graphically built $3 D$ environments, interact with digital artifacts and various functional tools and represent themselves through avatars, to communicate with other participants and engage in collaborative art learning. The goal is to establish an e-technology rich augmented art learning environment to create a more effective e-learning communities of practice.
\end{abstract}

Keywords: System architecture, Art Education, Virtual Reality, Presence

\section{Introduction}

Nowadays, virtual reality (VR) industry has been growing extensively that may lead ICT industry of next generation. Virtual reality refers to a computer-generated environment which provides viewers with visual illusion and sensation of being inside an artificial world that exists only in the computer. As coined by Lanier in 1989 in conjunction with his Virtual Programming Language Research [1], VR allows users to experience sensory input in a three-dimensional space [2]. With immersion technology, virtual reality creates a level of sensory fidelity which depends on measurable system attributes such as field of view (FOV), display size, stereoscopy, display resolution, head-tracking, or input devices [3].

VR system types can be categorized as non-immersive systems such as desktop computer systems, semi-immersive with large, multiple monitors that provide a medium to high level

Received (February 19, 2018), Review Result (May 2, 2018), Accepted (May 18, 2018)

*Corresponding Author 
of immersion, and fully immersive systems such as CAVE-style multiple screens and headmounted displays (HMD). The application of VR technology has significant potential in the field of education due to its functionalities useful to students understand complex data in science. VR spaces have been used in various areas such as art, science, medical science, and general education [4]. With the potential of virtual reality technologies, it is worthy to explore the uses of VR for educational purposes and seek possible uses in art education as well.

In many researches, VR technology takes up important role in education field and a research on art education is under progress. However, using VR for art education purposes has not been explored sufficiently and little is written on VR in art education compared to other educational applications of VR. It is possible to access the online virtual world and community and use it to help art educators open art classes in virtual environment. This technology has an advantage that the audience could visit, tour famous art hall or gallery virtually and by three-dimensionally visualizing art work based on curriculum of the teachers, such works could be shared and discussed with the students based on spatial information in existing plane information. VR technology could become a useful tool by which children could be familiar with a plane and spatial concept and by this technology, existing art work could be arranged in space. New technological tools for enhancing art teaching and learning process is emphasize the possibilities offered by arts education. Moreover, the use of VR for art educational purposes extends the realm of VR and may allow more people to see the important role of art in their lives.

In education, visual information is very important and a sense of immersion may differ depending on its features and resolution. Delivery level of visual sense was raised by expressing black and white image with single color and applying indefinite attribute to color image. Use of 3D image technology could raise a sense of space and reality by using binocular vision element in delivering a sense of space using just monocular vision element. In the past, 3D image equipment like CAVE and HMD were mainly used for research field but owing to development of computer hardware, 3D display system like HMD is being sold to general public [5]. As VR technology is used in game, relevant base technology has been developed and as low cost devices were sold, research range of VR technology has been extended from academic research stage to using stage of daily life. Due to this extension of use range, an attempt of applying virtual environment technology to education field has been increased and a research on possibility of immersion type display system has been progressed [6]. In addition, there are theoretical questions related to the design of models, methodologies and evaluation of VR systems that have hardly ever been addressed and studied in depth. Thus, this research is principally motivated by the non-existence of a theoretical foundation in the VR field and proposes a VR technology architecture for art education.

\section{Related Research}

The main features of virtual reality has been established by several researches such as the use of head-mounted displays and projection-based display [7, 8]. Various technologies that enabled the development of virtual reality converged to create the VR systems. Despite the development of virtual reality technology over a long period, VR has just begun to shift away from the theoretical and towards the practical [9] and VR system are starting to demonstrate practical effectiveness in real industrial settings [10]. The use of VR is predicted to broaden in telerobotics, telepresence surgery, virtual chemistry, virtual planetary travel, virtual entertainment, and architectural design. More than fifty VR applications in desktop and HMD environments for diverse educational purposes has been described [11], showing that the use of VR can be extremely widespread. VR can help students learn new concepts through VR technology which provides positive prospect of using VR in education. Several studies found that many of the children involved were able 
to learn VR through the process of creating virtual spaces [12].

3D image technology has an advantage that it could deliver a sense of space by providing 3-dimensional information, not a plane information. Visualization of 3D spatial information also acts as an important advantage in various education fields. Students majoring in architecture or machinery could design building or products by using 3D modeling tool like 3D CAD and confirm their outcome designed by 3D image system in a space. In the medical field, by using image scanner like CT, MRI, an analysis was performed by visualizing consecutive 2D images as 3D image. As human body organ location could be delivered more clearly if using 3D information rather than 2D one, 3D image has been used effectively in medical field and it was verified through several researches [13, 14].

In the art field also, a sense of space has been regarded as an important element from the past. In the paintings, perspective was used for showing a sense of space in a plane. What played a decisive role in order to express a sense of space is a linear perspective based on a geometric principle and perspective has been developed by many painters thereafter and it provided an opportunity of being able to make infinite space in canvas. 3D effect is created as image of human left, right eye is different and some painters tried to draw art works based on this basic 3D space concept. As shown on his art work, 'The sleeping smoker', Salvador Dali demonstrated art work showing features of binocular parallax of 3D image by drawing image of left, right eye differently. 'Osmose' of Charlotte Davies of Canada is an art work using HMD and it is composed of 12 spaces that analyzed an image of the nature symbolically and the audience experience a world different from reality while swimming around cyber space of their own volition. By using 3D image, omni-directional visual information that could be seen by the audience is provided and audience could be visually, deeply recognized and by re-structuring image of virtual environment as if the audience is moving by receiving movement information, spatial reality is made to be delivered [15].

Harmony Quest is a VR for an interdisciplinary art curriculum which was designed to expose students to VR technology in order to raise students' awareness of important roles in collaboration and to develop multidisciplinary curricula for the benefit of many people. Another goal was to make VR accessible in schools and communities. The use of Harmony Quest enabled students to extend their confidence in their own learning as they experienced VR within supportive groups. Through this teaching and learning process, students were exposed to diverse information, resources, Internet, e-mail in virtual environment.

Virtual environments evaluation has focused on evaluation of presence, which involves if how real or natural the user's experience was when immersed in the environment. Presence can be evaluated through the application of questionnaires which ask users to rate various qualities of the virtual environment ranging from perceptions of being there to more detailed inventories ranking controls, feedback, and perception of realism and user engagement $[16,17]$. Presence measures can benchmark virtual environment designs in terms of their realism and overall user experience.

\section{Virtual Reality System Architecture for Art Education}

This chapter will discuss the architecture of virtual reality system that will be applicable for art education. The virtual reality architecture for art education should be a useful reference framework for designing and implementing education programs that implement virtual reality as an educational technology. The virtual environments for art education support interaction between educators and learners and enable multiple simultaneous participants to access graphically built 3D environments, interact with digital artifacts and various functional tools and represent themselves through avatars, to communicate with other participants and engage in collaborative art learning. It is designed to provide an architecture that will cater the following goals.

First, the proposed architecture has the capability to be applied to any art learning 
program, irrespective of its complexity and its initial independence of external factors. Second, one of the main advantages of the model is their potential universality because it can be used in virtual environments as multisensory representations that can be experienced and interpreted by all students thereby facilitating teamwork and interaction between participants who are engaged into arts.

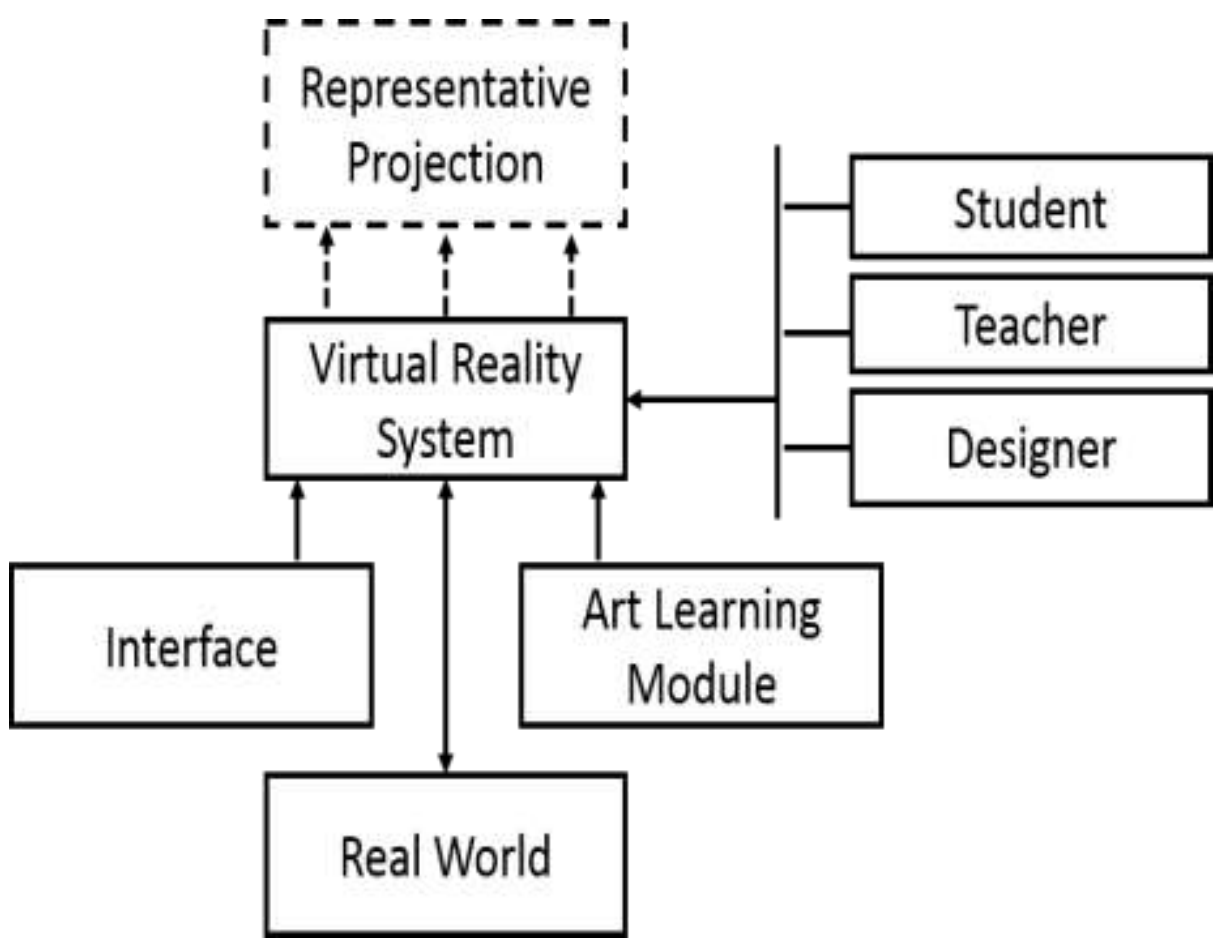

Figure 1. Virtual Reality Architecture for Art Learning

Figure 1 shows the proposed architecture proposed in this paper which identifies the essential components of the architecture of an educational system using virtual reality for art learning namely: modules, resources, processes and participants. Each component are described which includes the explanation of its main features and functionalities.

\subsection{Real World}

The real world can be defined as the setting in which learning of arts takes place. This setting must be taken into account when selecting and studying the knowledge sources. The educational environment can play a fundamental role and determine the forms of learning. Examples of real environments are painting room, music laboratory, observatory, the field, the street, the landscape, or the school classrooms. Thus, a key question is being raised that before designing a virtual reality art education system is to properly select the source knowledge for learning arts. Apparently, it makes no sense to teach all educational contents using this technology, either because they can be directly learn using traditional techniques or because different educational technologies are more effective and cheaper depending on the field of education.

This study establishes that abstract knowledge in arts is best suited for a virtual learning environment. Abstract knowledge means mainly complex concepts such as notions, theories, models, rules, processes and laws regarding art learning that have the following characteristics. First, they do not have a clear correspondence in the real world. As such, they are sometimes abstract, theoretical or generic entities, of which there are no references or specific examples in the real world. Second, the knowledge concerned is characterized as invisible, having no profiles or physical observations and cannot, therefore, be 
experienced in practice or perceived by the human senses. Moreover, they are ideal in general. Thus, abstract objects and cognitive processes that are difficult to visualize or imagine, concepts that are complicated to represent graphically or explain verbally.

It is viewed that the teaching and learning of this kind of knowledge sources raises enormous difficulties in traditional education. Selecting abstract contents, like source knowledge, will help to better identify what unique features virtual reality has as a pedagogical tool. In addition, another issue that arises is how to represent and teach abstract concepts in art learning in a virtual environment. In an artificial world, primarily based on physical or sensory experience, scientific cognition will certainly call for new and original forms of symbolization and representation.

\subsection{Representative Projection}

Virtual reality always provides a representative parallel to the real world. The architecture designed for the virtual reality system utilizes the fundamental role of representation to design, structure, and building of meaningful virtual reality systems. The learning of source knowledge can be made easier by its representative embodiment in virtual worlds, where students can visualize, experience and interpret this knowledge directly to the art learning.

The key component of the proposed framework is the representative projection, which can be defined as a mapping between the source knowledge of the real world and the virtual world. The main goal of this process of representational transfer is to build a system or network of representations capable of defining the structure of the virtual world and organizing how to learn, navigate and interact. Thus, representative projection takes place on four different interdependent planes namely: learning plane, interaction plane, structural plane and navigation plane. Table 1 provides a summary of the different planes in representative projection of the proposed virtual reality system architecture.

Table 1. Different Planes of Representative Projection

\begin{tabular}{|c|l|}
\hline Plane & \multicolumn{1}{c|}{ Goal } \\
\hline Learning plane & Design the pedagogical approach and strategies \\
\hline Interaction plane & Establish how learners interact with the virtual scenarios \\
\hline Structural plane & $\begin{array}{l}\text { Create a representation to provide isomorphism between real } \\
\text { world and virtual world } \\
\text { Provides how students browse and move around in the virtual } \\
\text { world }\end{array}$ \\
\hline Navigation plane
\end{tabular}

One of the most important components of the representative projection is the learning planes which largely determine the selection of the representation in the other planes. The learning plane representation serve to design the pedagogical approach and strategies which are the activities open to art students in the virtual world, the role of student and teacher, the representation or symbolization of the didactic contents in the virtual scenarios. The representation of the interaction plane establish how students can interact with the virtual scenario, how they manipulate the objects they come across and how they communicate with other possible participants. Another significant component of representative projection is the structural plane. The structural plane is composed of the representation that create the isomorphism between the source knowledge and the virtual world. These representation establish the organizational principles of the virtual scenario, determining its form and its structure. The aim is to base and structure the source knowledge in a familiar domain or situation that is already understood by students. The isomorphism or structural similarity will help them to discover the central features of the source knowledge domain. The 
navigation plane describes how users browse and move around in the virtual world such as walking, driving, and using a transportation tool. The representation of this plane also define the scale or reference framework in which users move, as well as the students' viewpoint.

These four planes make up what should be a systematic and consistent set or network of representation. The representative projection is an indispensable tool for conceiving the preliminary design of the two primary facets of the virtual world. Firstly, the design of the architecture of the virtual scenario: the structural and learning planes define both the configuration of the elements that constitute the structure of the virtual world, such as their symbolic meaning (the embodiment or metaphorical representation of the contents of learning in this physical space). Secondly, the design of how the student can inhabit or use the environment: the navigation and interaction plane describe the different forms in which the user can move around and interact with objects and other virtual world participants (or how the user can interpret the meaning of the scenario).

\subsection{Virtual Reality System}

A virtual reality system is a computer application capable of generating a 3D environment in which the user is an active participant and interacts with the artificial world using a range of interfaces. According to [18-20], the main features offered by virtual worlds are as follows:

- Presence. In a virtual reality system, users experience the feeling that they are actually there in the real place. Students are carried off to an environment of pure information that they can see, listen to and touch. In virtual worlds where presence is high, the sensation of immersion is so strong that the interface disappears and users lose all notion of interacting with a computer system.

- Autonomy. A virtual environment is autonomous and dynamic when it is capable of pursuing its own goals, executing actions and evolving, irrespective of user interactions.

- Navigation. Users can be either immobile observers or travelers in the virtual environment, moving around in different ways such as walking, saying, speaking, using a vehicle, touching an object or pointing in any direction within the environment.

- Interaction. Users can make use of a range of ways of manipulating and modifying virtual worlds. Students could move the virtual objects by hand, eye movement or voice. Also, they have the ability to create and alter the environment.

- Scale. The scale of the virtual environments can be altered, changing the relative size of users in respect of the virtual world and allowing students to become the same size as the biggest thing such as a star or the smallest object.

- Viewpoint. This provides the possibility of users changing perspective at will. For example, students could pass on their viewpoint to a given artificial world object or process, or even to the viewpoint of another participant. Students can also be a floating viewpoint, saying or moving at any speed in any direction.

- Co-operative learning. Distributed and networked environments provide for collective participation, offering several users the possibility of sharing virtual spaces at the same time. The real-time interaction between different students leads to genuine co-operative learning accordingly. 


\subsubsection{Virtual Reality System Components}

The virtual reality applications for art education are classified using input and output media for the virtual world simulation. A user is placed in a real-time simulation and immersed in a world that can be both autonomous and responsive to its actions.

\subsubsection{Input}

For art education, virtual reality technology input medium are defined as something that allows humans to give out information and interact with the environment. People interact with the world mainly through locomotion and manipulation and convey information by means of voice, gestures, and facial expressions [21]. Communication using gestures as well as locomotion make full body motion analysis desirable, while verbal communication with the computer or other users makes voice input an important option. It is possible to limit user input to a few selected channels. As the hand offers many more degrees of freedom concentrated in a small area than any other part of the body, hand motion tracking is sufficient for most applications. Moreover, the fact that the hand is the privileged manipulation tool makes hand motion tracking a critical input for interacting with virtual worlds. Viewpoint specification requires real time motion tracking of the user's head, and possibly eyes, in order to update displayed stereo images in coordination with user movements.

\subsubsection{Output}

The output medium of a virtual reality application correspond to human senses namely: touch and force perception, vision, hearing, smell and taste. Perception is based on the interpretation of signals sent to the brain by the human senses which affects how a person react to the world and how one interpret events in his/her surroundings. As such, sensory simulation is at the heart of virtual reality technology.

- Position. Touch and Force Perception. The haptic sense is capable of both sensing what it is happening around the human being and acting on the environment. This makes it an indispensable part of many human activities and thus, in order to provide the realism needed for effective applications, VR systems need to provide inputs to, and mirror the outputs of, the haptic system. The primary input/output variables for the haptic sense are displacements and forces.

- Visual Perception. Vision is generally considered the most dominant sense, and there is evidence that human cognition is oriented around vision [22]. High quality visual representation is thus critical for virtual environments. The major aspects of the visual perception that have an impact on display requirements are the depth perception, accuracy and field-of-view and critical fusion frequency.

- Sound Perception. Hearing is mainly used for verbal communication in order to get information from invisible parts of the world or when vision does not provide enough information. Audio feedback must be able to synthesize sound, to position sound sources in 3D space and can be linked to a speech generator for verbal communication with the computer. In humans, the auditory apparatus is most efficient between 1000 and 4000 $\mathrm{Hz}$, with a drop in efficiency as the sound frequency becomes higher or lower [23].

- Olfactory Perception. Specialized applications exist where olfactory perception is of importance. One of these is surgical simulation, which need to provide the proper olfactory stimuli at the appropriate moments during the procedure. Similarly, the training of emergency medical personnel operating in the field should bring them into 
contact with the odors that would make the simulated environment seem more real and which might provide diagnostic information about the injuries that simulated casualty is supposed to have incurred [24]. A VR environment giving olfactory cues should provide the possibility to diffuse the odors when needed and purify and filter the air when the cue is no longer required.

The requirements in terms of input and output medium has highlighted performance requirements for the simulation of existence of synthetic objects. Successful virtual reality applications must combine new input and output devices in ways that provide not only such an illusion of existence of synthetic objects, but also the interaction metaphors for interacting with them.

In addition, augmented reality technology includes a technology of being able to augment virtual information based on real world. By this features, children could receive 3D image technology naturally. In education curriculum for the children who are not familiar with digital device, a method of having children show interest in art education is useful by adding one's own existing idea rather than generally completing a new art work through VR technology [25]. In case that art education is combined with game element, student usually show more concern [26]. VR technology has an advantage that it could be used for exercise method for producing art work and a new world is structured and an activity is performed in it [27]. Art work is required to have a form being harmonized with special environment based on intention of the painter. Students could cultivate an ability of understanding and analyzing visual environment in VR world.

\subsection{Art Learning Module}

The proposed architecture uses art learning module which are designed to perform the following functions. Firstly, it should provide the capability to select and clearly determine the source knowledge that are to be taught. The possible influence of the real environment on art learning also needs to be examined. Secondly, there should be a way of establishing the student profile: age, level of background knowledge, whether or not they have any physical or cognitive impairment involving special educational needs, their previous experience with virtual reality systems, as well as any other characteristic possibly influencing learning. Thirdly, art students can choose a given pedagogical program or learning theory, such as cognitivism, constructivism, the neo-Deweyist paradigm, the theories of Piaget, etc., as a guide for the learning process. These are the functions of any learning module to be applied in a real environment. The following function, however, is specific to education in virtual worlds. Finally, there is a need to implement a preliminary design of the virtual world by means of a representative projection, creating a meaningful isomorphism between the knowledge of the real environment and the virtual world for art students. This preliminary design will serve to establish how the didactic material should be presented and structured, as well as to define the role of students and teachers in learning art in the virtual world. Those who are in charge of the learning module are teachers and educators with elementary knowledge of the rudiments of virtual reality technology, as well as the framework of this architecture.

\subsection{Participants}

The participants included in the virtual reality system architecture are the designers, students, and teachers. The term designers refers to a wide-ranging group of professionals responsible for carrying out the final virtual reality system design and implementation. Designers are comprised of analysts, mechanical, electrical, optical and sound engineers, graphic designers, and computer programmers. Analysts who are responsible for designing high-level virtual world hardware and software. It is their responsibility to assure that the different planes of the representative projection are consistent and systematic. In addition, 
they need to check that it is possible to implement the model as it has been designed. Mechanical, electrical, optical and sound engineers to design and construct the multisensory interfaces and devices of the virtual reality system. Graphic designers to create the 3D models of the virtual scenario and animations. Computer programmers to write the software to control the complex simulations.

Users are students and teachers who are inhabiting and experiencing the virtual environment through different interfaces or input and output devices, such as head-mounted displays, gloves, body suits, trackers, 3D sound systems, etc., which provide the sense of immersion through visual, haptic, kinesthetic and auditory feedback. Both teachers and students could be participant in design work if they have enough knowledge about designing the virtual reality architecture. Teachers with advanced knowledge of virtual reality technology can make a direct contribution to the final system design and implementation.

\subsection{Design Criteria for Art Education}

In the learning style of the proposed architecture, the art instructional design must begin from the pedagogical support of the specific environment. Teachers should design their classes to the appropriate degree of difficulty, stimulate creativity, encourage knowledge sharing, develop case studies, and enable the establishment of evaluation criteria. Educators we have to know that the field of art corresponds to a profound human desire as an exceptional presence.

\section{Evaluating the VR System for Art Education}

Evaluating the VR system can be achieved with the involvement of arts education teachers. The arts education teachers selects the art learning modules related to visual communication and multimedia technology. The art learning modules comprise of fundamentals on pedagogical use of ICT in education, e-learning technologies and learning platforms, visual communication and multimedia technology in education, and teaching methodologies and pedagogical strategies based on the use of virtual instrumentation. These include designing learning activities which include the use of virtual experiments in education. The art learning module is compulsory for the entire target group, the participants has the option to choose the function of their needs and preferences.

The art learning modules that have been developed in a blended learning format such as face-to-face and e-learning, different aspects have been presented and discussed. The most important of those were how to implement new technologies in education, in strong relation with the use of available computers and other hardware specific devices or how to use the software in teaching and learning process and transform it in additional didactic instruments with educational valences.

A questionnaire can be applied to the target group in order to collect the teachers' opinion related to the aspects presented during the courses. Some of the questionnaire items can be devoted to obtain the teachers' feedback related to the expected benefits of using ICT in the teaching / learning process. As shown in Table 2, it present the trainees' perception on how the ICT tools such as multimedia products can increase the scientific content understanding, improve the teaching methods in arts classes, attract the learners to study and cooperate or increase the students' self-motivation. A five-level Likert scale can be used for evaluating each aspect. Most of the arts teachers agrees that multimedia products like digital presentations, educational video-clips, electronic posters or sound files have $a$ great potential to support the Arts education. These kinds of ICT tools can help only to some extent the teaching/learning approach in terms of improving the teaching methods or increasing the scientific content understanding. 
Table 2. Trainees Perception Questions

\begin{tabular}{|l|}
\hline \multicolumn{1}{|c|}{ Questionnaire Items } \\
\hline Increase scientific content understanding \\
\hline Improve teaching methods \\
\hline Attract learners to study and cooperate \\
\hline Increase self-motivation \\
\hline
\end{tabular}

\section{Conclusion}

A system architecture of VR technology on art education was researched and its relevance with art theory and application case were explored. In a perspective that VR technology is a special media that may deliver a sense of space, its convergence with art in which a sense of space is applied through perspective was analyzed to be effective. Direct interaction technology that could be seen in media art recently shows very close relation with VR technology. When observing relevant technology, in art field also, VR technology could provide high reality and emotional information and induce higher immersion level, its possibility in art education is represented. In case that an education is progressed based on VR technology by targeting students having concern over art, a lot of concern and interest could be provided. However, as merit, demerit by application of a new technology coexist, if the teachers should apply such elements to the students by fully understanding it, VR technology would be settled down as an effective education method. Quality evaluation model of VR technology being linked with empirical test knowhow in art education in which real time 3D environment build-up technology that may borrow methodology defining requirement of major features at quality requirement definition stage based on international standard and matrix that could be expressed at evaluation preparation stage and implement VR is accumulated will be researched in the future.

\section{Acknowledgments}

This work was supported by the National Research Foundation of Korea (NRF) grant funded by the Korea government (No. NRF-2017R1D1A1B030 34041)

\section{References}

[1] J. M. Albani and D. I. Lee, "Virtual reality-assisted robotic surgery simulation", Journal of Endourology, vol. 21, no. 3, (2007), pp. 285-287.

[2] K. Sakatani, "Harmony quest: An interdisciplinary arts-based project incorporating virtual reality", In Delacruz (Ed.), Visual Art Research, Champaign, IL: Visual Arts Research, vol. 31, (2005), pp. 53-62.

[3] D. A. Bowman and R. P. McMahan, "Virtual Reality: How Much Immersion is Enough?", Computer, vol. 40, (2007), pp. 36-42.

[4] P. A. M. Kommers and Z. Zhiming, "Virtual reality for education. University of Twente, East China Normal University", Retrieved December 6, 2007, from http://projects.edte.utwente.nl/proo/kommers.htm, (1997).

[5] T. B. Sheridan, "Teleoperation, telerobotics and telepresence: A progress report", Control Engineering Practice, vol. 3, no. 2, (1995), pp. 205-214.

[6] M. Roussou, "Immersive interactive virtual reality and informal education", Proceedings of User Interfaces for All: Interactive Learning Environments for Children, (2000).

[7] I. E. Sutherland, "A head-mounted three dimensional display", In Proc. the Fall Joint Computer Conference, (1968), pp. 757-764.

[8] M. W. Kruger, "Responsive environments", In NCC Proceedings, (1977), pp. 375-385.

[9] F. Biocca, "Communication within virtual reality: Creating a space for research", Journal of Communication, vol. 42, no. 4, (1992), pp. 5-22.

[10] C. Youngblut, R. E. Johnson, S. H. Nash, R. A. Wienchclaw and C. A. Will, "Review of virtual environment interface technology", IDA Paper P-3186, Institute for Defense Analysesc, (1995).

[11] C. Youngblut, "Educational uses of virtual reality technology", Alexandria, VA: Institute for Defense Analysis, (1998). 
[12] C. Byrne, "Virtual reality and education", In proceedings of IFIP WG3.5 International workshop conference, (1993).

[13] N. W. John, "The impact of Web3D technologies on medical education and training", Computers \& Education, vol. 49, no. 1, (2007), pp. 19-31.

[14] R. Coulter, "The effect of degree of immersion upon learning performance in virtual reality simulations for medical education”, Medicine Meets Virtual Reality, vol. 15, (2007).

[15] C. Davies and J. Harrison, "Osmose: towards broadening the aesthetics of virtual reality", (1996).

[16] M. Slater, V. Linakis, M. Usoh and R. Kooper, "Immersion, presence and performance in virtual environments: an experiment using tri-dimensional chess", Available online at http://www.cs.ucl.ac.uk/staff/m.slater/Papers/Chess/index.html, (1996).

[17] B. G. Witmer and M. J. Singer, "Measuring presence in virtual environments: a presence questionnaire", Presence, vol. 7, (1999), pp. 225-240.

[18] M. Bricken, "Virtual worlds: No interface to design", In Cyberspace: First steps, M. Benedikt (ed), MIT Press, Cambridge, MA, (1991).

[19] C. M. Byrne, "Water on tap: The Use of Virtual Reality as an Educational Tool", Ph. D. Dissertation, Department of Industrial Engineering, University of Washington, Seattle, WA, (1996).

[20] D. Zeltzer, "Autonomy, interaction and presence", Presence, vol. 1, (1992), pp. 127-132.

[21] J. Balaguer and A. Mangili, "Virtual environments", In New Trends in Animation and Visualization., D. Thalmann and N. Magnenat-Thalmann, Eds. Wiley, New York, NY, USA, (1992).

[22] S. Kosslyn, "Image and Brain: The resolution of the imagery debate", MIT Press, Cambridge, MA, USA, (1994).

[23] C. Youngblut, R. E. Johnson, S. H. Nash, R. A. Wienchclaw and C. A. Will, "Review of virtual environment interface technology", IDA Paper P-3186, Institute for Defense Analysesc, (1995).

[24] M. W. Krueger, "Olfactory stimuli in virtual reality for medical applications", In Interactive Technology and the New Paradigm for Healthcare, (1995), pp. 180-81

[25] Y. Huang, H. Li and R. Fong, "Using Augmented Reality in early art education: a case study in Hong Kong kindergarten”, Early Child Development and Care, vol. 186, no. 6, (2016), pp. 879-894.

[26] K. Copič Pucihar, M. Kljun and P. Coulton, "Playing with the artworks: engaging with art through an augmented reality game", Proceedings of the 2016 CHI Conference Extended Abstracts on Human Factors in Computing Systems, ACM, (2016).

[27] H.-C. Han, "Teaching Visual Learning through Virtual World: Why Do We Need a Virtual World for Art Education?", Art Education, vol. 68, no. 6, (2015), pp. 22-27. 
International Journal of Advanced Science and Technology

Vol.114 (2018) 